\title{
Structure Preserving Data Abstractions for Statecharts
}

\author{
Steffen Helke and Florian Kammüller \\ Technische Universität Berlin, \\ Institut für Softwaretechnik und Theoretische Informatik
}

\begin{abstract}
Hierarchical automata (HAs) represent a structured model of statecharts previously formalized in Isabelle/HOL. The present work extends this framework by an abstraction technique for HAs defined on infinite data spaces. This structure preserving abstraction enables the connection of the framework to the model checker SMV. This paper reports on the following results (a) We discuss abstractions of sequential automata, from which HAs are composed. Here we focus on the special problems of synchronous models and examine the feasibility of constructions for over- and underapproximations in order to preserve CTL properties. (b) Based on this results we describe a compositional abstraction technique, which can be applied to HAs. (c) We extend the formalization of HAs in Isabelle/HOL by suitable operators to construct abstractions inside the logic. (d) We present an efficient implementation of the abstraction process outside of the logic, which is integrated in the formalization by the oracle interface of Isabelle.
\end{abstract}

\section{Introduction}

In earlier work we already proposed a formalization of Hierarchical Automata (HAs) [KH00,HK01] in Isabelle/HOL [Pau94]. The motivation for this project has been, and still is, to provide a mechanized support for statecharts [HN96]. As Mikk [Mikk00] has already correctly observed, a formal treatment of statecharts needs an ameliorated calculus with an improved hierarchical structure that facilitates the analysis of (a) the typical hierarchical states of statecharts and (b) the consequently intricate structure of inter-level transitions. In the formalism of HAs these obvious problems can nicely be resolved. Therefore, we have adopted the HAs as well as a basis for the formalization in Isabelle/HOL.

The second major goal of the formalization of statecharts is to provide mechanized support for statecharts containing data. Therefore, in addition to the original formalization [KH00,HK01] we have furthermore suggested an extension in which finite HAs, i.e. HAs containing only finite data can already be verified by a connected model checker SMV efficiently [HK03]. However, the crux with data contained in a state transition model like statecharts or HAs, respectively, is that the state space can become infinite. Hence, the ultimate goal of the formalization of statecharts in Isabelle/HOL that has now been achieved has been 
to integrate the formalization with the well-known technique of abstract interpretation [CC77] in order to make any data containing statecharts amenable to automatic verification with the support of a connected model checker. To enable reasoning in the formalization at the concrete and abstract level and to improve readability we devise an abstraction in which the result of the abstraction is again a hierarchical automaton.

The basic idea of the integration of Isabelle/HOL with the SMV model checker (and in principle any model checker that is suitably adjusted to check finite HAs) is to host the HAs in an Isabelle/HOL embedding and control the abstraction process there. The connection to the model checker is realized via Isabelle's oracle interface. Although in principle feasible - as shown in this paper - to express a general abstraction operator based on Galois connections inside the Isabelle/HOL model, we provide in addition for practical purposes an MLimplementation for the construction of the HA abstraction.

The most advanced result presented in this paper is the construction of an abstraction process for infinite HAs. The techniques used in the abstraction are based on earlier work [SS99,Dam96], but we go further than that as we do not consider just simple flat transition systems but realistic hierarchical state transition models as they are used in software engineering. For example, UML integrates state-machines that are basically the same as statecharts. Moreover in contrast to [SS99] the abstraction in the formalization inside Isabelle is not restricted to just boolean abstractions. Finally, this formalization is complemented with an implementation following the outline of [SS99] but lifted to hierarchical automata. The resulting integrated framework including model checking is better suited for handling case studies.

\section{Abstraction of Sequential Automata}

In [HK01,Mikk00] Sequential Automata (SAs) are the basic building block of HAs. They are similar to simple transition systems. The syntactic structure of an HA is described as a tree-like structure containing the SAs. This syntactic representation of HAs is complemented by a semantical model in our framework. Each level of hierarchy of an HA is represented by one SA.

In this section we propose a property preserving abstraction technique for SA, that are defined on infinite data spaces. The result of the abstraction is again an $\mathrm{SA}$. In the next section we reuse this basic theory to explain the compositional abstraction for HAs.

\subsection{Property Preservation and Galois Connections}

The framework of [HK01] includes a formalization of CTL [CE81], which is interpreted on HAs and on SAs respectively. In the present work, we investigate property preserving abstraction for SAs in order to preserve CTL formulas. In the literature we can find basically two approaches: over- and underapproximation. 


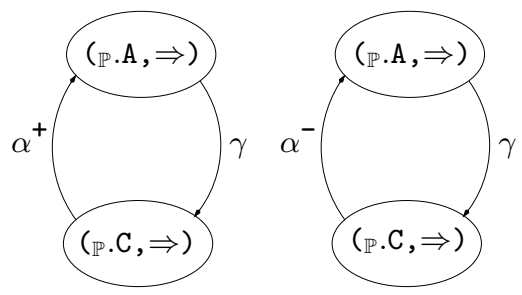

Fig. 1. Galois Connections for Over- and Underapproximation of Predicates

In an overapproximation the abstract model can contain new behaviour, but old behaviour cannot be lost. That is, properties of the universal fragment of $C T L(\forall C T L)$, that are valid on all paths of the overapproximated abstract model, must hold on the paths of the concrete model. In contrast, in an underapproximation new behaviour cannot be added, but old behaviour can be lost. Accordingly properties of the existential fragment of $C T L(\exists C T L)$ are preserved by underapproximated abstract models. In the work of Dams [Dam96] two automata representing these different kinds of abstractions are generated. Depending on the property that has to be verified the appropriate model has to be chosen.

In recent work [HJS01] the information for over- and underapproximation is represented in one so called modal transition system by may and must transitions. For verifying these transition systems special model checkers are proposed. Compared to a traditional symbolic model checker like SMV [McM93] such tools are inefficient. Moreover in our framework we like to generate SAs as abstractions, whose semantics is defined on traditional transition systems. Consequently, we chose to base our framework on the work of Dams and adapt his abstraction process to SAs. We must restrict the process to overapproximations, because SAs will not allow a reduction by underapproximation. Details are explained in the following subsection.

The theoretical basis for over- and underapproximations are galois connections [MSS86]. For complete lattices, $\mathrm{C}$ and $\mathrm{A}$, a pair of monotone maps, $\alpha: \mathrm{C} \rightarrow \mathrm{A}$ and $\gamma: \mathrm{A} \rightarrow \mathrm{C}$ define a galois connection, written gc $(\mathrm{A}, \mathrm{C}, \alpha, \gamma)$, iff $\alpha \circ \gamma \leq i d_{\mathrm{A}}$ and $i d_{C} \leq \gamma \circ \alpha$. Furthermore the maps in a galois connection satisfy the following adjunction theorems, which allows to define $\alpha$ by $\gamma$ and vice versa using the generalized meet and join $(\bigvee, \wedge)$ that exist in complete lattices.

$$
\begin{aligned}
\alpha \cdot \mathrm{c} & =\bigwedge\{\mathrm{a}: \mathrm{A} \mid \mathrm{c} \leq \gamma \cdot \mathrm{a}\} \\
\gamma \cdot \mathrm{a} & =\bigvee\{\mathrm{c}: \mathrm{C} \mid \alpha \cdot \mathrm{c} \leq \mathrm{a}\}
\end{aligned}
$$

Figure 1 represents two instantiated galois connections relating spaces of abstract and concrete predicates. Accordingly the elements are ordered by $\Rightarrow$. On the left hand side of the figure we define a galois connection gc ( $\left.\mathbb{P} . \mathrm{A}, \mathbb{P} . \mathrm{C}, \alpha^{+}, \gamma\right)$ for overapproximation to weaken concrete predicates by the abstraction $\alpha^{+}$, which is reflected in the following galois property.

$$
\mathrm{p}_{\mathrm{c}} \Rightarrow \gamma \cdot \alpha^{+} \cdot \mathrm{p}_{\mathrm{c}}
$$



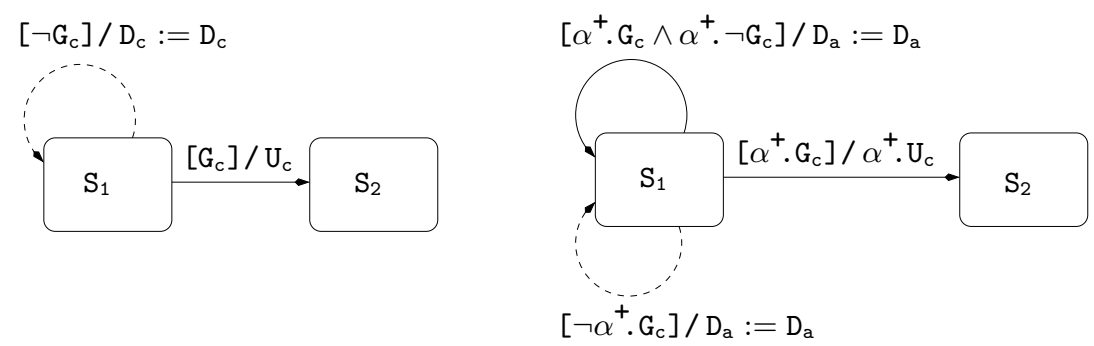

Fig. 2. Implicit Behaviour and Overapproximation of an SA

Correspondingly on the right hand side, a galois connection gc ( $\mathbb{P} \cdot \mathrm{C}, \mathbb{P} \cdot \mathrm{A}, \gamma, \alpha^{-}$) for underapproximation is given, in order to strengthen concrete predicates by the abstraction $\alpha^{-}$, which is reflected in the following galois property.

$$
\gamma \cdot \alpha^{-} \cdot \mathrm{p}_{\mathrm{c}} \Rightarrow \mathrm{p}_{\mathrm{c}}
$$

A special effect is, that overapproximations can be expressed by underapproximations.

$$
\alpha^{+} \cdot \neg \mathrm{p}_{\mathrm{c}} \Leftrightarrow \neg \alpha^{-} \cdot \mathrm{p}_{\mathrm{c}}
$$

We use this property for the definition of the construction operators and also to reduce the complexity in the ML implementation of an abstraction algorithm. Furthermore, we are going to use the fact that the strongest postcondition SP and the weakest precondition WP form a galois connection gc ( $\mathbb{P} . A, \mathbb{P} . C, S P, W P)$ to define the construction operators in Section 4.

The ML implementation of our abstraction technique is a predicate abstraction similar to the work of [SS99]. Usually the abstraction function $\alpha$ is defined for predicate abstractions by $\gamma$ using the adjunction theorems (see above). We need this property in Section 5 .

\subsection{Generating Overapproximations for SAs}

This paper is based on a formalization of statecharts as HAs [HK01,HK03]. It includes a formalization of SAs. The semantics of SAs is there a special case of the semantics of HAs, because SAs can be viewed as HAs without hierarchy. Based on this formalization we introduce an abstraction technique for overapproximation of SAs. Statecharts and consequently HAs - as the communication principles stay the same - belong to the family of synchronous languages. So the used formalization as HAs and SAs reflects the properties of synchronous languages and has to be respected in the abstraction process. One special property of synchronous languages is, that in each semantical status - synchronized by a global clock - the system performs a defined calculating step. Semantical statuses of SAs where no transitions fire, perform a trivial calculating step, in which the data variables are assigned to the previous value. This effect can be interpreted as complementation by implicit transitions. 
On the left hand side in Figure 2 this is depicted by a dashed self-transition, where the guard $\neg \mathrm{G}_{\mathrm{c}}$ is constructed as the negated guard of the exiting transition. In general, this guard must be constructed as the conjunction of negated guards of all exiting transitions. Overapproximating an SA we construct an identical structured SA. We adopt the control states and abstract the transitions. Abstracting transitions we abstract guards and updates separately.

In general it is impossible to construct a guard in the abstraction by the given predicates exactly. Firstly, we propose to weaken a guard $\mathrm{G}_{c}$ by $\alpha^{+}$using overapproximation. Building such weaker guard adds new behaviour to the model, however it deletes some implicit behaviour simultaneously caused by the special semantics of synchronous languages. The reason is, that the guard of the implicit transition $\neg \alpha^{+}$. $\mathrm{G}_{\mathrm{c}}$ will be automatically stronger. Therefore, secondly we must add a suitable self transition, to adjust this unwanted effect. The guard of this self transition must be constructed by a conjunction of the overapproximated guard of $\mathrm{G}_{\mathrm{c}}$ and the negated underapproximated guards of all exiting transitions. On the right hand side of Figure 2 this procedure is illustrated.

Abstracting the example only one exiting transition has to be considered. Firstly, we abstract the guard $\mathrm{G}_{\mathrm{c}}$ by $\alpha^{+}$and introduce a self-transition. The guard of this self-transition is constructed by a conjunction of the overapproximated guard of the exiting transition $\alpha^{+}$. $\mathrm{G}_{\mathrm{c}}$ and the negation of the underapproximated guard of the exiting transition $\neg \alpha^{-}$. $\mathrm{G}_{\mathrm{c}}$. The latter can be expressed by $\alpha^{+}$using the theorem of subsection 2.1, so that finally we obtain the following guard for the self-transition.

$$
\left[\alpha^{+} \cdot \mathrm{G}_{\mathrm{c}} \wedge \alpha^{+} \cdot \neg \mathrm{G}_{\mathrm{c}}\right]
$$

Building traditional overapproximation of updates [SS99] is compatible with SAs, because a weaker update adds new behaviour to the system, but old behaviour cannot be lost. Accordingly on the right hand side of Figure 2 the update is overapproximated by $\alpha^{+}$. However, in general, abstracting updates results in non-constructive predicates, so that the action language of SAs is violated. More precisely for each $\mathrm{U}_{\mathrm{c}}$ we obtain in general more than one abstract update by $\alpha^{+}$.

We must restrict to overapproximation, because SAs will not allow a reduction by underapproximation. This is caused by the special semantics of synchronous languages, which can be interpreted as a complementation by implicit transitions. This complementation restricts the possibility for reduction of the behaviour fundamentally. Consider the example in Figure 2 on the left hand side. If we propose to build a stronger guard of $\mathrm{G}_{\mathrm{c}}$ by $\alpha^{-}$, we obtain a weaker guard for the implicit transition consequently. That is, we add new behaviour to the abstract model, which is unsound for underapproximation. Hence, for the example on the left hand side of Figure 2 we cannot build an underapproxmiation, where the abstract model is again an SA. The only way out is to reduce nondeterministic branches to deterministic ones, however this is not sufficient as a general procedure. Consequently the result of an underapproximation cannot usually be expressed by an SA. 


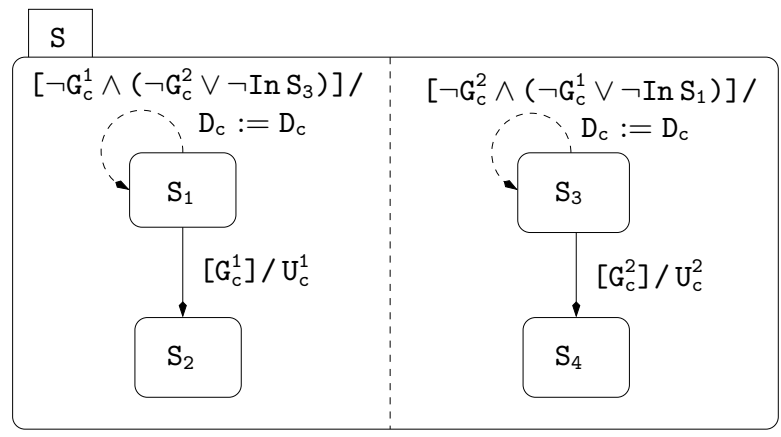

Fig. 3. Implicit Concrete Behaviour of HAs

\section{Abstraction of Hierarchical Automata}

In this section we introduce the lifting of the abstraction process to the level of HA. A HA is constructed from a finite set of SAs and a composition function $\mathrm{CF}$ that describes the hierarchical relation between those SAs. The first subsection 3.1 gives a brief introduction to semantical characteristics of HAs. In the following subsection we describe how we can handle global information of update-function locally by a generic parameter. The last subsection presents a structure preserving overapproximation for HAs.

\subsection{Semantical Characteristics of HAs}

The literature contains several accounts addressing the rather complex semantics of statecharts [HN96,Mikk00]. For a better understanding of the abstraction theory the interpretation of implicit behaviour as well as the partitioning of the data space are introduced in some detail as they play a central role for abstraction.

Implicit Concrete Behaviour: Implicit behaviour occurs in synchronous modelling languages whenever a transition cannot fire at the beginning of a clock cycle. In this situation the statechart executes a trivial calculation step that restores the data state. In Section 2 the implicit behaviour has been represented in the model by a dashed arrow (cf. Figure 2). This special transition only fires if no other transition of the model is enabled. As is shown in Figure 3 we can model the implicit behaviour of a HA explicitly in a similar fashion. Note, however, that with respect to compositional abstraction, the guard of the self-transition depends on context information that lies outside the SA in which the self-loop is defined. Considering the SA on the left hand side of the Figure, we observe that the guard of the implicit self-transition of the control state $\mathrm{S}_{1}$ holds, if and only if the guard $G_{c}^{1}$ of the transition exiting $S_{1}$ does not hold. In 
addition a predicate of the parallelly composed SA must hold. Either the control state $S_{3}$ is not active or the guard of the transition exiting $S_{3}$ is not valid. More generally, in all parallelly composed SAs there must not be any transition that is enabled.

The modelling of implicit behaviour of HAs shown in Figure 3 may become complicated, because usually we have more than one local state in an SA. The concept of generic update functions - presented in the next subsection - can avoid this effect, because they abstract from the dependencies between parallelly composed SAs (cf. Figure 4). Additionally generic update functions can model partial updates, which is not supported by the modelling of Figure 3.

Partitions on Data Spaces and Partial Update-Functions: In general, the data space of an HA consists of a finite number of disjoint partitions. Updatefunctions can be defined in such a way that they do not write on all partitions. The semantics of HAs determines the values of partitions after transition execution also in cases in which a transition does not write on the partition.

That is, the update-functions are partial. More precisely, in one step of calculation of HA transitions of several SAs that are composed in parallel can be executed synchronously. If a transition does not write on a partition, it is first examined whether there is another synchronously executed transition writing on this partition. If this is the case, the value of the synchronously executed transition is selected. In case of a concurring write of several transitions on one partition (so-called racing) the resulting conflict is resolved by introducing a non-determinism (interleaving semantics). In contrast, if there is no transition writing on a partition, the semantics assigns to this partition the value prior to execution of the transition.

Note, that there already exists a complete formal semantics of HAs including data spaces in Isabelle/HOL [HK03]. Furthermore there the idea of generic update-functions is presented, which we will introduce in the next subsection.

\subsection{Generic Update-Functions in an HA-Context}

When considering SAs as constituents of an HA the individual data spaces of the SA have to be embedded into the global data space of the HA. There are two entities of an SA and its abstraction that are influenced by this embedding: the update-functions given in the action parts of the transitions of an SA and the self-loops that are added to the states of the SA during the abstraction. The embedding of an SA is simply given by adding an additional context parameter to the update-functions of the SA representing the remainder of the global data space. The effect of the update-functions is extended in the embedding by explicitly assigning those context parameter to their pre-state. We call these update-function generic as they abstract over context information and can thus be used for arbitrary contexts.

In Figure 4 we see the left SA of Figure 3. Differing from Figure 3 we used a generic update-function here. In this example we assume that the data space is 


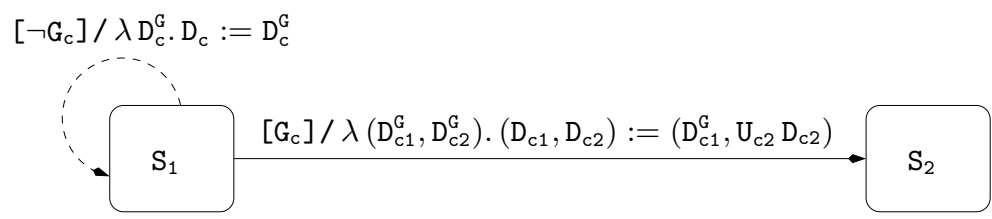

Fig. 4. Implicit Behaviour of SAs Including a Generic Update-Function

divided into two partitions represented by the Cartesian product ${ }^{1}$. We further assume that the update-function writes just on the second partition $\mathrm{D}_{\mathrm{c} 2}$ using the update-function $\mathrm{U}_{\mathrm{c} 2}$. The update-function $\mathrm{U}_{\mathrm{c} 2}$ works on partitions and is derived from an update-function $U_{c}$ that operates on the entire data space. The generic update-function abstracts over a context parameter $\left(D_{c 1}^{G}, D_{c 2}^{G}\right)$ that has a partitioning identical to the data space. The generic parameter $D_{c 1}^{G}$ is passed on to the first partition of the data space. Here, the well-formedness of the updatefunction requires that $D_{c 1}^{G}$ must not be modified as this would lead to a kind of micro-step semantics that we do not intend.

Generic update-functions may be employed similarly for the description of implicit behaviour. In Figure 4 we use in contrast to Figure 3 a modified label to annotate the dashed self-transition. Using a generic update-function the guard can be weakened and may now be defined only using the information $\neg \mathrm{G}_{\mathrm{c}}$ locally available in the SA. The self-transition cannot describe more implicit behaviour, because the reachable data states in the post state coincide with data states of possibly synchronously firing transitions due to the generic parameter of the update-function.

When introducing the overapproximations for SAs in the Section 2 we assumed that update-functions always write on all partitions of the data space. The aim of the next subsection 3.3 is to adapt the given abstraction concept to generic update-functions that do not write on the entire data space, but only some of its partitions.

\subsection{Overapproximations for HAs}

The idea for the abstraction of the HA is to divide the HA into its defining SAs, define the abstraction functions for each of the SAs and compose the set of individual abstractions to an abstraction function for the HA.

The resulting abstraction method for HA preserves properties of the concrete $\mathrm{HA}$ in the constructed abstract HA because it respects the structure of the HA and the constituting SAs are adjusted prior to composition in such a way that they are properly embedded into the data space of the HA.

The latter step - realized by an extension of each update-function of an SA by a context parameter as explained in the last subsection - allows to abstract

\footnotetext{
${ }^{1}$ In our Isabelle theory of abstraction partitions are realized as a list of sum types (cf. Section 4).
} 


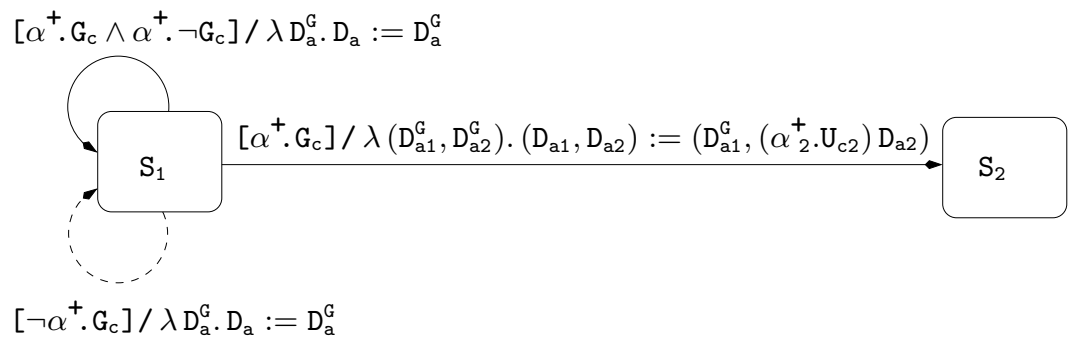

Fig. 5. Abstraction of SAs Including a Generic Update-Function

SAs separately. However we need a technique for dealing with the generic context parameters.

We suggest two possibilities for the abstraction of generic update-functions.

In the first naïve approach the generic context parameter of the updatefunction is not preserved during abstraction. This abstraction is rather rough. It assumes that in partitions, on which locally no write occurs, to which the context parameter has been passed on, arbitrary behaviour may occur. In Section 4 we define an operator that constructs such an abstraction.

The elaborated approach to the abstraction of generic update-functions is to preserve the generic parameter during abstraction. This is only possible if the given abstraction-function preserves the structure of the data space and each data partition of the concrete system is mapped independently of other partitions onto exactly one partition of the abstract data space. Figure 5 shows such an abstraction for the example introduced in Figure 4 . In this example the concrete generic parameters $\left(D_{c 1}^{G}, D_{c 2}^{G}\right)$ are replaced by corresponding abstract representations $\left(D_{a 1}^{G}, D_{a 2}^{G}\right)$. The update-function $U_{c 2}$ can be overapproximated by

$\alpha_{2}^{+}$. Note, however, that $\alpha_{2}^{+}$can only be calculated precisely if the abstraction function respects, as described above, the structure of the data space. Otherwise the elaborated approach is not applicable and we have to use again the naïve alternative. Furthermore, $\alpha_{2}^{+}$calculates in general more than one abstract update-function.

\section{Calculating Abstractions in Isabelle}

The concepts presented in the previous section have been transformed into a theory of abstraction for the theorem prover Isabelle. This new theory extends the existing theory of HAs by calculating operators that enable the construction of an abstract HA from a concrete HA and an abstraction function.

\subsection{Calculating Overapproximated SAs}

In order to construct the abstraction of an SA inside the logic, we define in Isabelle/HOL (suitable) calculating operators that - given a concrete $\mathrm{SA} \mathrm{SA}_{\mathrm{c}}$ and 
an abstraction function $\mathrm{R}$ - construct an abstract SA using overapproximation. The construction operator $\mathrm{AbsBy}_{\mathrm{SA}}^{+}$is defined in Isabelle as follows.

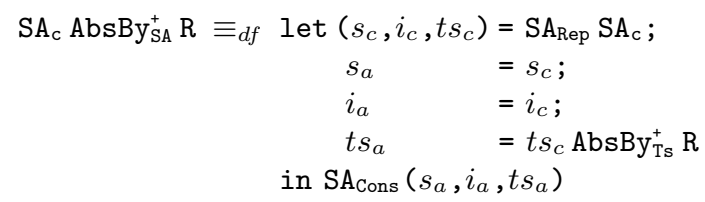

First the concrete $\mathrm{SA} \mathrm{SA}_{\mathrm{c}}$ is transformed into its representation. Thereby its concrete components, like the set of control states $s_{c}$, the initial set of control states $i_{c}$ and the transition relation $t s_{c}$ become accessible. Since we want to construct a structure preserving abstraction for the SA, we adopt the structural information from $s_{c}$ and $i_{c}$ as they are.

Abstracting the transition relation by the construction operator $\mathrm{AbsBy}_{\mathrm{Ts}}^{+}$each transition of $t s_{c}$ is abstracted separately. According to the presented procedure in Figure 2 we abstract a transition $t_{c}$ in two steps. Firstly, we build an abstract transition replacing guard and update-function of $t_{c}$ by corresponding overapproximated counterparts. Secondly, we generate a self transition on the source state of $t_{c}$, which is labeled by the negation of the underapproximated guard of $t_{c}$ and an update-function, that assigns the previous value to the data variables. Note, that the construction of the self transition could be more precise, because we ignore the guards of other exiting transitions in the source state of $t_{c}$. The core of the construction lies on one side in the abstraction of the guard with the operator $\mathrm{AbsBy}_{\mathrm{G}}^{+}$and on the other side in the abstraction of the updatefunction with the operator $\mathrm{AbsBy}_{\mathrm{U}}^{+}$. The following constant definition introduces the construction operator for the abstraction of a guard.

$\mathrm{G}_{\mathrm{c}} \mathrm{AbsBy}_{\mathrm{G}}^{+} \mathrm{R} \equiv_{d f} \lambda d_{a} \cdot \exists d_{c} \cdot\left(\mathrm{G}_{c} d_{c}\right) \wedge\left(\mathrm{R} d_{c}\right)=d_{a}$

The definition corresponds to a strongest postcondition and weakens the property $\mathrm{G}_{\mathrm{c}}$. The weakening can be proved as the following theorem. The proof is by stepwise simplification.

$$
\begin{aligned}
&\left(\mathrm{G}_{\mathrm{c}} d_{c}\right) \Rightarrow\left(\mathrm{G}_{\mathrm{c}} \mathrm{AbsBy}+\mathrm{G}\right)\left(\mathrm{R} d_{c}\right) \\
& \Leftrightarrow \quad\left(\mathrm{G}_{\mathrm{c}} d_{c}\right) \Rightarrow \exists d .\left(\mathrm{G}_{c} d\right) \wedge(\mathrm{R} d)=\left(\mathrm{R} d_{c}\right) \\
& \Leftrightarrow \quad\left(\mathrm{G}_{\mathrm{c}} d_{c}\right) \Rightarrow\left(\mathrm{G}_{\mathrm{c}} d_{c}\right) \wedge\left(\mathrm{R} d_{c}\right)=\left(\mathrm{R} d_{c}\right)
\end{aligned}
$$

In addition to the overapproximation of a guard we need to calculate the overapproximation of an update-function. First we consider the formal description of an operator that defines such an abstraction.

$$
\mathrm{U}_{\mathrm{c}} \mathrm{AbsBy}_{\mathrm{U}}^{+} \mathrm{R} \equiv_{d f}\left\{\mathrm{U}_{\mathrm{a}} \cdot \forall d_{a} \cdot \exists d_{c} \cdot d_{a}=\left(\mathrm{R} d_{c}\right) \wedge \mathrm{R}\left(\mathrm{U}_{\mathrm{c}} d_{c}\right)=\mathrm{U}_{\mathrm{a}}\left(\mathrm{R} d_{c}\right)\right\}
$$

The result of this overapproximation of a concrete update-function $\mathrm{U}_{\mathrm{c}}$ is a finite set of abstract update-functions containing at least one element. The definition given above simulates the behaviour of an abstract update-function by the behaviour of a concrete update-function. Figure 6 illustrates graphically the encoded simulation property. Similar to the overapproximation of guards, the abstraction of an update-function may as well be described using the strongest 


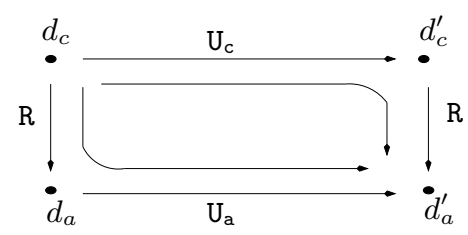

Fig. 6. Simulation Property for Abstraction of Update-Functions

postcondition. To this end, update-functions are interpreted as binary predicates defined over the pre-states and post-states of the data. Such a predicate may be derived directly from an update-function U using the operator UP.

$$
\mathrm{UPU} \equiv \equiv_{d f} \lambda d d^{\prime} \cdot d^{\prime}=(\mathrm{U} d)
$$

The operator AbsBy ${ }_{\mathrm{UP}}^{+}$defines how the abstraction of a concrete update-function $\mathrm{U}_{\mathrm{c}}$ can be expressed as an abstract binary predicate

$$
\mathrm{U}_{\mathrm{c}} \mathrm{AbsBy}_{\mathrm{UP}}^{+} \mathrm{R} \equiv \equiv_{d f} \lambda d_{a} d_{a}^{\prime} . \exists d_{c} d_{c}^{\prime} .\left(\mathrm{U}_{\mathrm{c}} d_{c}\right)=d_{c}^{\prime} \wedge\left(\mathrm{R} d_{c}\right)=d_{a} \wedge\left(\mathrm{R} d_{c}^{\prime}\right)=d_{a}^{\prime}
$$

The fact that the operators $\mathrm{AbsBy}_{\mathrm{U}}^{+}$and $\mathrm{AbsBy}_{\mathrm{UP}}^{+}$describe equivalent abstract behaviour is proved in Isabelle by the following theorem.

$$
\operatorname{Surj} R \Rightarrow\left(U_{c} A_{b s B y}^{+} R=\bigvee U P '\left(U_{c} A_{U P s B y}^{+} R\right)\right)
$$

This equivalence holds under the assumption that the abstraction function $\mathrm{R}$ is surjective. To be able to compare the operators with each other we first transform the set of abstract update-functions in a set of abstract binary predicates using the operator UP. Finally, the disjunction of all those predicates is equivalent to the predicate constructed using the operator $\mathrm{AbsBy}_{\mathrm{UP}}^{+}$.

\subsection{Calculating Overapproximated HAs}

In order to provide a construction for HAs as well, we reuse the operator $\mathrm{AbsBy}_{\mathrm{SA}}^{+}$ introduced in the previous section and extended corresponding to Figure 5. The operator $\mathrm{AbsBy}_{\mathrm{HA}}^{+}$implements this idea as follows.

$$
\begin{aligned}
& \mathrm{HA}_{c} \mathrm{AbsBy}_{\mathrm{HA}}^{+} \mathrm{R} \equiv_{d f} \text { let }\left(d_{c}, s a s_{c}, e s_{c}, c f_{c}\right)=\mathrm{HA}_{\mathrm{Rep}} \mathrm{HA}_{c} ; \\
&=\mathrm{R} d_{c} ; \\
&=\left(\lambda s a_{c} \cdot s a_{c} \mathrm{AbsBy}_{\mathrm{SA}}^{+} \mathrm{R}\right){ }^{\prime} \mathrm{SA}_{c} ; \\
&=e s_{c} ; \\
& e s_{a}=c f_{c} \\
& c f_{a} \\
& \text { in } \mathrm{HA}_{\mathrm{Cons}}\left(d_{a}, s a s_{a}, e s_{a}, c f_{a}\right)
\end{aligned}
$$

First we transform the concrete $\mathrm{HA} \mathrm{HA}_{\mathrm{c}}$ into its representation to gain access to the concrete components, like the initial data state $d_{c}$, the finite set of SAs $s a s_{c}$, the set of events $e s_{c}$, and the composition function $c f_{c}$. Since we want to construct a structure preserving abstraction for the HA, we adopt the structural information of $e s_{c}$ and $c f_{c}$ as they are. In contrast, the two other components 
that involve data, have to be abstracted in a suitable fashion. To this end we abstract the initial data state of the HA using the abstraction-function R. To abstract the SAs the operator $\mathrm{AbsBy}_{\mathrm{SA}}^{+}$is applied to each element of the set $\operatorname{sas}_{c}$. Note, however, that the operator $\mathrm{AbsBy}_{\mathrm{SA}}^{+}$has been adapted accordingly to enable in addition the abstraction of partial update-functions. The abstraction of partial update-functions is now discussed in more detail.

An update-function is a function that calculates a new data value from the data value prior to execution of a transition. This leads to the following polymorphic type definition in the Isabelle/HOL formalization.

$\delta$ update $\equiv_{d f} \delta \rightarrow \delta$

As described in Section 3 the polymorphic type $\delta^{2}$ may consist of finitely many disjoint partitions. Furthermore, update-functions can only write on specific partitions of the global data space. In Isabelle/HOL all functions are total. Therefore, we had to develop an appropriate model of updates on partitions on which no write is performed that respects the statecharts semantics. To this end we extend the type of the update-function by an additional data parameter. That is, we define a kind of generic update-function as follows.

$$
\delta \text { update } \equiv_{d f}[\delta, \delta] \rightarrow \delta
$$

Wellformedness of the update-function requires that the values of a partition contained in a generic parameter may only be passed on but must not be altered. In the semantics of HAs this parameter may be instantiated and evaluated by the context information that is then available. In this step it is checked whether synchronously firing transitions write on the partition. If there is no write, the old value that has been valid prior to the transition's execution is assigned. In addition we define an resolution for write conflicts (racing) using non-determinism in the semantics. A precise semantical foundation for update-functions is provided by earlier work [HK03].

The problem with compositional abstraction is that, in general, only local information is available, and therefore a technique is needed to abstract a generic update-function. For the abstraction of generic update-functions it is important to define a solution in which the abstract update-function is also well-formed. We suggest two solutions.

The first naïve solution does not permit generic parameters in an abstract update-function. When calculating abstract updates the post-state of any data partition may only depend on locally determined data values. Information passed by the generic data parameter may not be used for the calculation of the poststate. This may, in certain cases, lead to a rather rough approximation. More precisely, if we ignore the generic parameter for the abstraction, we get in a first step a set of concrete non-generic update-functions describing the possible effects.

Updates $\mathrm{UG}_{\mathrm{c}} \equiv_{d f}\left\{\mathrm{U}_{\mathrm{c}} \cdot \exists d_{c}^{g} \cdot \mathrm{U}_{\mathrm{c}}=\left(\lambda d_{c}^{p} \cdot \mathrm{UG}_{\mathrm{c}} d_{c}^{g} d_{c}^{p}\right)\right\}$

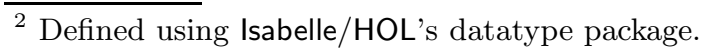


The following constant definition $\mathrm{AbsBy}_{\mathrm{UG}}^{+}$constructs from this set of updatefunctions a set of non-generic abstract update-functions.

$$
\mathrm{UG}_{\mathrm{c}} \mathrm{AbsBy}_{\mathrm{UG}}^{+} \mathrm{R} \equiv_{d f} \bigcup\left(\left(\lambda u_{c} \cdot u_{c} \mathrm{AbsBy}_{\mathrm{U}}^{+} \mathrm{R}\right) \text { '(Updates } \mathrm{UG}_{\mathrm{c}}\right) \text { ) }
$$

The naïve approach is feasible in cases where all concrete update functions write on the entire data space, because in this case the operator Updates yields just one function. However, in cases where generic update-functions write only on parts of the data space, the naïve approach leads to approximations that lack precision.

The presentation of the formalization of the elaborated solution is omitted here, but we give an outline. This approach requires that the abstraction function $\mathrm{R}$ is constructed such that for each partition there is a suitable abstraction that maps this partition independent of other partitions onto an abstract data partition. On one side, this procedure preserves the structure of the data space. On the other side, it enables the simulation of the behaviour of concrete generic update-functions by the behaviour of abstract generic update-functions. To this end, the previous polymorphic type $\delta$ is refined into a list of sum-types where each element of the list describes a partition of the data space. Based on this structured data-type, we can formalize the requirement on the abstraction function stated above and define a corresponding operator for the construction of generic update-functions.

\section{Implementation of the Abstraction Process}

Besides the Isabelle definitions of operators for the construction of overapproximated HAs from a given HA and an abstraction function, we have in addition implemented an abstraction algorithm that is connected via the so-called oracleinterface of Isabelle to our Isabelle theory. This algorithm has been developed as a diploma thesis. It adapts the algorithm for predicate abstraction of transition systems suggested by Shankar and Saïdi [SS99] to HAs. In this kind of abstraction data variables of a transition system, that are declared on infinite data domains, are interpreted abstractly by a finite number of characteristic predicates. Adapting this algorithm to HAs for each partition of the data space predicates will be defined independently. These predicates must be defined by an expert and will be given as an input to the abstraction process. In the construction of the abstract data space, concrete data variables on infinite domains are replaced by a finite number of boolean variables. Each boolean variable encodes whether a corresponding characteristic predicate is satisfied or not. Accordingly the concretization function $\gamma$ of the galois connections for predicate abstraction is given by a substitution, that replaces in an abstract formula the boolean variables by the corresponding predicates. Usually the abstraction function $\alpha$ is defined by $\gamma$ using the adjunction theorems (cf. Section 2). So we obtain for over- and underapproximation the following abstraction functions.

$$
\begin{aligned}
& \alpha^{+} \cdot p_{c}=\bigwedge\left\{p_{a}: \mathbb{P} \cdot A \mid p_{c} \Rightarrow \gamma \cdot p_{a}\right\} \\
& \alpha^{-} \cdot p_{c}=\bigvee\left\{p_{a}: \mathbb{P} \cdot A \mid \gamma \cdot p_{a} \Rightarrow p_{c}\right\}
\end{aligned}
$$


In practice it is not feasible to calculate all formulas of $\mathbb{P}$.A. So we must restrict ourselves to representative formulas. [SS99] proves that it is sufficient for calculating an overapproximation, to show $\mathrm{p}_{\mathrm{c}} \Rightarrow \gamma \cdot \mathrm{p}_{\mathrm{a}}$ for all disjunctions, that can build on the introduced boolean variables. So the complexity can be reduced to at most $3^{k}-1$ proof obligations, where $k$ represents the number of boolean variables used to represent the predicates.

As a result of the implementation we found that already for small examples the number of abstract update functions calculated for one concrete updatefunction is rather high. This problem can be controlled if the predicates used for the abstract interpretation of a partition are chosen such that they are mutually exclusive and do not overlap.

\section{Conclusions}

In this paper we have presented a concept for the abstraction of statecharts containing data. The concept is based on a formalization in the generic interactive theorem prover Isabelle/HOL. Following the approach of [Mikk00], we use a representation of statecharts as hierarchical automata. The methodology of building the abstraction is similar to earlier work on abstract interpretation of transition systems by Cousot and Dams. However, the novelty here is that the existing concepts are transferred to the formalism of statecharts that (a) enable to structure the state space and (b) contain data. While there is some work on mechanical analysis of transition systems containing data using abstraction techniques [MN95] and some mechanizations of statecharts, e.g. [BW98], we are not aware of any work dedicated to the statecharts formalism combining theorem proving and model checking.

The embedding in the theorem prover represents a semantical foundation for data enriched statecharts enables the calculation of overapproximations, and serves well as a logical framework for mechanically proved abstractions. We have developed a structure preserving abstraction technique for HAs that can be applied in a compositional manner. To this end we extended the abstraction theory for transition systems to SAs. To keep our method compositional we use a novel technique that respects context informations. For the analysis of case studies we integrated an implementation of an algorithm for the abstraction process outside Isabelle.

In contrast to other approaches of abstraction theories for model checking we do not yet consider a complementary refinement process that is used to refine too rough abstractions in order to regain better approximations. Currently we plan to adapt our approach for detecting spurious counterexamples [CGJ $\left.{ }^{+} 00\right]$ to refine the abstraction in a suitable way.

Acknowledgment. We would like to thank Dr. J.Sanders from PRG Oxford for his support and comments. This work has been partly supported by the DFG/BC ARC project. 


\section{References}

[BW98] U. Brockmeyer and G. Wittich. Tamagotchis need not die - verification of statemate design. In B. Steffen, editors, Tools and Algorithms for the Construction and Analysis of Systems, TACAS'98, Springer LNCS, 1384, 1998.

[CC77] P. Cousot and R. Cousot. Abstract Interpretation: A Unified Lattice Model for Static Analysis of Programs by Construction of Approximation of Fixed Points. In Proceedings of the 4th ACM Symposium on Principles of Programming Languages, ACM, New York, 1977.

[CE81] E. M. Clarke and E.A. Emerson. Synthesis of synchronization skeletons for branching time temporal logic. In D. Kozens, editor, Logic of Programs: Workshop, Springer LNCS, 131, 1981.

$\left[\mathrm{CGJ}^{+} 00\right]$ E. Clarke, O. Grumberg, S. Jha, Y. Lu and H. Veit. Counterexample-guided Abstraction Refinement. In A.P. Sistla, E.A. Emerson, editors, Computer Aided Verification, CAV00, Springer LNCS, 1855, 2000.

[Day93] N. Day. A model checker for statecharts. Technical Report TR-93-35, Department of Computer Science, University of British Columbia, October 1993.

[Dam96] D. Dams. Abstract Interpretation and Partition Refinement for Model Checking. PhD thesis, Eindhoven University of Technology, Netherlands, July 1996.

[HN96] D. Harel and D. Naamad. A STATEMATE semantics for statecharts. ACM Transactions on Software Engineering and Methodology, 5(4):293-333, Oct 1996.

[KH00] F. Kammüller and S. Helke. Mechanical Analysis of UML State Machines and Class Diagrams. In Defining Precise Semantics for UML, Sophia Antipolis, France, June 2000. Satellite Workshop, ECOOP 2000.

[HK03] S. Helke and F. Kammüller, Verification of Statecharts Including Data Spaces. In D. Basin, B. Wolff, editors, TPHOLs 2003: Emerging Trends Proceedings, Technical Report Albert-Ludwigs-Universitat Freiburg 189, 2003.

[HK01] S Helke and F. Kammüller. Representing Hierarchical Automata in Interactive Theorem Provers. In R. J. Boulton, P. B. Jackson, editors, Theorem Proving in Higher Order Logics, TPHOLs 2001, Springer LNCS, 2152, 2001.

[HJS01] M. Huth, R. Jagadeesan and D. Schmidt. Modal transition systems: a foundation for three-valued program analysis., editors, European Symposium on Programming, ESOP 2001, Springer LNCS, 2028, 2001.

[McM93] K. McMillan. Symbolic Model Checking: An Approach to the State Explosion Problem. Kluwer Academic Publishers, 1993.

[Mikk00] E. Mikk. Semantics and Verification of Statecharts. PhD thesis, Christian Albrechts Universität Kiel, 2000.

[MN95] O. Müller and T. Nipkow. Combining Model Checking and Deduction for I/O Automata. In TACAS95, 1st International Workshop on Tools and Algorithms for the Construction and Analysis of Systems, Springer LNCS, 1019:116, 1995.

[MSS86] A. Melton, D.A. Schmidt and G. . Strecker. Galois connections and Computer Science applications, Springer LNCS, 240:299-312, 1986.

[Pau94] L. C. Paulson. Isabelle: A Generic Theorem Prover, Springer LNCS, 828, 1994.

[SS99] H. Saïdi and N. Shankar. Abstract and Model Check While You Prove. In N. Halbwachs and D. Peled, editors, 11th Interantional Conference on Computer Aided Verification, CAV99, Springer LNCS, 1633, 1999. 